\title{
Risques mineurs, changements majeurs
}

Minor risks, major changes

\section{Elizabeth Gardère et Nicole d'Almeida}

\section{CpenEdition}

\section{Journals}

Édition électronique

URL : http://journals.openedition.org/communicationorganisation/4456

DOI : 10.4000/communicationorganisation.4456

ISSN : $1775-3546$

\section{Éditeur}

Presses universitaires de Bordeaux

\section{Édition imprimée}

Date de publication : 1 juin 2014

Pagination : 1-16

ISBN : $978-2-86781-904-9$

ISSN : $1168-5549$

\section{Référence électronique}

Elizabeth Gardère et Nicole d'Almeida, "Risques mineurs, changements majeurs », Communication et organisation [En ligne], 45 | 2014, mis en ligne le 27 octobre 2014, consulté le 22 septembre 2020. URL : http://journals.openedition.org/communicationorganisation/4456 ; DOI : https://doi.org/ 10.4000/communicationorganisation.4456 


\title{
Risques mineurs, changements majeurs
}

\author{
Élizabeth Gordère ${ }^{1}$ et Nicole D'Almeida ${ }^{2}$
}

L'objectif de ce numéro est d'analyser comment s'opère, sous l'effet des dispositifs d'information et de communication, le couplage entre une culture généralisée du risque et une réorganisation du social, des pratiques et des modes de vie tant individuels que collectifs. À la différence de bon nombre de publications consacrées aux crises et risques dits majeurs, nous avons souhaité nous inscrire dans un horizon qui n'est ni celui de la catastrophe, ni celui de l'apocalypse, mais dans la perspective du vécu et du quotidien. Si un certain nombre de travaux invitent à penser que des événements majeurs n'ont guère produit de changement majeur dans les pratiques ultérieures (de Tchernobyl à Fukushima), nous avons décidé de nous pencher sur l'ordinaire du changement, lieu souterrain où s'opèrent de puissantes transformations. Consacré à des objets minuscules (telles les nanotechnologies) ou mineurs (l'e-cigarette, le menu de la cantine), explorant les lieux de la quotidienneté (l'école, l'hôpital, la table familiale ou l'entreprise), ce numéro ne se résout pas à la force des choses mais explore la dynamique sociale, humaine et communicationnelle en présence et la puissance de transformation en jeu.

Dans le travail de qualification-configuration de la catégorie de risque est en jeu un processus civilisationnel de l'ordre de ce qu'étudiait Norbert Elias dans La Civilisation des Mours. La généralisation de la question du risque désormais présent dans tous les interstices de la vie avive la tension entre une idéologie libertaire de l'ouverture et de la créativité et une idéologie sécuritaire et normalisée qui anime tous les aspects de la vie quotidienne : consommation de produits et de services, relations aux autres, fonctionnement des organisations, attentes envers les institutions. La recherche affichée d'une société de l'innovation s'accompagne paradoxalement d'une volonté de risque zéro et de la création de dispositifs de normalisation organisés à de vastes échelles. Une même tension anime l'espace discussionnel fondamentalement

1 Professeure des Universités - Université de Bordeaux. MICA (EA 4426) - Université Bordeaux Montaigne ; elizabeth.gardere@u-bordeaux.fr

2 Professeure des Universités - Celsa Paris - Sorbonne. GRIPIC - ISCC ; Nicole.d'almeida@celsa.parissorbonne.fr 
polémique $^{3}$ du risque, marqué par l'articulation entre le saillant et le lisse, entre les aspérités du vécu de crises renouvelées et le lissage des discours préventifs, explicatifs construits à l'intention de destinataires virtuels dont est présupposée la capacité rationnelle et prudentielle. Le risque fonctionne comme un ferment communicationnel, comme un potentiel discussionnel qu'actualisent des groupes nombreux dans un éventail ouvert de thèmes, comme si une relecture complète de notre société pouvait se faire à partir de la catégorie de risque. La technosphère semble bien ici étroitement liée à la logosphère. La question du risque n'est pas une question objectivable uniquement par des chiffres et des calculs statistiques, elle ne peut à l'inverse être campée dans une perspective totalement constructiviste qui assimilerait le risque à un être de langage. Entre perspective probabiliste et spéculative, la question du risque renvoie à des modèles culturels de représentations, à un imaginaire social remodelé à l'heure de la globalisation, technicisation et complexification. Le caractère central et ambigu du risque l'installe au cœur de relations renouvelées entre les sphères scientifique, médiatique, politique et sociale. La généralisation du risque installe plus fortement la science en société, convoque scientifiques et experts dans l'espace de la société civile et des médias, la situe aux côtés du politique qui délibère et légifère. La question du risque n'est ni strictement technique, ni statistique, elle est une question totale qui engage la production de la société elle-même.

Inscrit dans la foulée de travaux menés dans diverses disciplines et poursuivant les acquis d'études menées en SIC, ce numéro fait rebondir la thématique du risque.

La question du statut du risque ne cesse de se complexifier ce qui est signe non pas de faiblesse mais de force : le développement du savoir gagne par là en vigueur, plusieurs lectures étant à la fois en présence et en tension. Ce faisant, l'approche traditionnellement statistique du risque permettant de qualifier le risque comme un aléa inscrit dans une série mathématique de probabilités reste la clé de voûte du système assurantiel et bancaire. Cette manière d'objectiver le risque (fondée sur un principe de spéculation mathématique) s'est ensuite complexifiée dans une société industrialisée, animée par unidéal de progrès dont le risque n'est pas absent mais dont la présence est située de manière résiduelle, comme à la marge. La perspective contemporaine s'inscrit en rupture avec ces considérations dès lors qu'elle situe le risque non pas à la périphérie mais au cœur des sociétés. Ce qui était conçu comme un aléa devient un point central, identifié en premier lieu par la pensée allemande. Les travaux de Habermas, Beck et Luhmann sont, sur ce point, pionniers et posent la question du risque comme expérience vécue et réfléchie, porteuse de changement majeur dans la fabrique du social et du politique. La visée des auteurs n'est pas unifiée,

3 A. Bouzon dans un article paru sur ce sujet in Communication $\mathcal{E}^{\circ}$ Organisation, n ${ }^{\circ} 20,2001$, p. 27-48, rappelait opportunément la double filiation étymologique possible du terme de risque renvoyé à resecum - l'écueil - ou à rixare - se quereller. 
invitant à penser les conditions de possibilité d'une activité de jugement et de délibération dans laquelle l'espace public gagne en extension, ce que ne manqueront pas de débattre les auteurs de ce numéro 45. À partir de la vision habermassienne revue à travers le cas des nanotechnologies, la thèse du délitement annoncé de l'espace public sous l'effet des forces du marché est interrogée au travers d'objets techniques minuscules et invisibles. Le second angle qui structure ce numéro thématique concerne l'articulation des niveaux individuel et collectif où se joue la question du risque dans son volet pratique, pédagogique et communicationnel. Problème collectif structurant les sociétés, le risque est, dans une société libérale, renvoyé à une affaire individuelle, à une affaire d'individus qu'il convient d'informer et de former. La tension est forte entre la perspective collective du risque, fait social total pourrait-on dire en reprenant l'expression que Marcel Mauss appliquait au don, et la perspective individualisante renvoyant à la fiction d'un individu libéral souverain, maître de lui et de ses choix. Maîtriser les risques liés à la consommation des aliments, au choix de l'eau à boire ou de la cigarette à fumer ou ne pas fumer est devenu une injonction contemporaine, une manière de situer la responsabilité et d'envisager de nouvelles techniques de soi selon l'expression de Foucault. Considérer le risque à l'aune de l'individualisation et envisager les problèmes ou contradictions suscités, telle est la vocation de plusieurs articles rassemblés dans ce numéro. De ce point de vue, le risque est évacué dans la mesure où les dimensions scientifiques, gestionnaires et politiques des risques collectifs industriels et technologiques, et leurs externalités négatives, ne seraient pas en cause. Dans ces produits nouveaux qui conjugueraient respect de soi et respect d'autrui, il n'y a ni expertise ni pragmatisme à la question du risque, mais plutôt une régulation et une normalisation du risque face à l'individualisme, voire à sa privatisation. La clef communicationnelle serait-elle celle de la responsabilisation d'acteurs autonomes face à leur prise de décision, liberté d'action et niveau d'information dans une société qui conjuguerait idéalement care et self control ? Partant du principe selon lequel les situations à risque sont le fait "d'un réseau d'interactions socioculturelles ", les auteurs mettent à l'épreuve les supports pédagogiques et informatifs issus des établissements scolaires destinés aux élèves et leurs parents. Les logiques d'acteurs en cause sont tripartites. Il s'agit du rôle des programmes ministériels, des médiateurs que sont les établissements scolaires ainsi qu'une entreprise et de l'appropriation faite par les destinataires. L'objectif est d'analyser la construction d'une relation de confiance sachant que la temporalité varie et peut, potentiellement altérer les retombées escomptées, voire la crédibilité de l'action. Le dénominateur commun de ces campagnes d'information est fait de stéréotypes et mises en scène caricaturales ou bien, a contrario, rebutantes, visant à inspirer dégoût, rejet ou moquerie des abus et dangerosités des produits alimentaires concernés. 
Entre la question collective du risque et le niveau individuel interviennent des médiations humaines ou techniques : lanceurs d'alerte ou dispositifs analysés ici dans deux articles. Déclencher ou barrer la voie, telle est la configuration envisagée dans ces textes. Tandis que l'espace public est de plus en plus structuré par la jurisprudence et la médiatisation, on peut dérouler le fil du cycle de vie du risque, de l'émetteur au destinataire et convoquer la notion de crise mise en scène sous la rampe des enjeux médiatiques. Qui lance l'alerte et à qui profite le risque ? Telles sont les questions posées. Les champs de la communication, de la sociologie et de la science politique sont mobilisés dans cet article autour du point crucial du lanceur d'alerte, leader d'opinion. Sachant que l'espace numérique est devenu le prolongement, le lieu privilégié de propagation du «virus » risque qui se répand dans les réseaux, les dynamiques changent, les leviers d'action et instruments du pouvoir aussi. On peut ainsi se demander quelles prérogatives restent à l'appareil institutionnel normatif traditionnel, aux circuits internes dans une société civile où la crédibilité de l'émetteur n'est plus nécessairement un critère (préventif, palliatif ou correctif).

Le troisième angle structurant ce numéro est celui de l'extension des champs du risque, extension observable à travers ces organisations variées analysées par les auteurs du numéro. La question du risque physique en organisation à laquelle une réponse a été donnée (en son temps) par la création des CHSCT et par les progrès de l'ergonomie et des démarches de qualité se double de la question du risque psycho-social, objet de drames et d'une législation plus récente. Le risque en organisation a pris la forme historique du risque professionnel sur lequel une législation spécifique a été déployée à la fin du $19^{\mathrm{e}}$ siècle (loi de 1898 sur les accidents de travail). Du risque en organisation, nous sommes passés à l'organisation comme risque, les entreprises étant considérées comme responsables de leurs actes et de leurs produits, ayant à répondre de leurs produits et de leurs processus (du mode de fabrication au mode de management). Elles se renouvellent selon des formes émergentes, numériques, interactives, dématérialisées, voire nomades. Il apparaît dans un article ici consacré à l'hôpital que si pour les soignants la tâche est peu impactée, les acteurs du management sont concernés.

Enfin, il nous a semblé important de situer la question du risque dans une perspective plus large où ne cessent de se renforcer les dimensions juridique et communicationnelle. La force du droit s'impose dans ce domaine, la dimension juridique s'est intensifiée au cours des années 1980, moment de développement d'une politique de prévention et de médiatisation des risques naturels et technologiques exercée au niveau juridique et sociétal. Si l'aléa et la conjonction sont les facteurs clefs du risque, la maitrise des deux par des outils de gestion et d'actions de prévention permet de réduire le niveau de risque, et donc de minimiser le degré de catastrophe potentielle. Ce renforcement des dispositifs de lois et de réglementations peut être renvoyé à un esprit des lois, 
à un esprit du temps, repère d'une « reconfiguration de l'imaginaire » forgée au cours des siècles dans les pays européens. Dans l'imaginaire contemporain, la communication, la participation et l'initiative citoyennes occupent une place de choix. Contrairement à la vision veloutée et pacifiée à laquelle nous invite l'appellation tant invoquée de société du safe et société du care, ce numéro de Communication E Organisation inscrit la question du risque dans la perspective d'une société d'énonciation et de dénonciation.

Une société d'énonciation dans laquelle l'omniprésence du risque serait jugulée par une prolifération de discours d'injonction, d'explication, de formation destinés à des publics supposés rationnels. Imaginaire d'un public, imaginaire de la force de l'information et de la communication sont ici fortement et tacitement mobilisés.

Une société de dénonciation dans laquelle le risque est un facteur d'interpellation, un moteur (de) critique générant de la parole et du débat, invitant à une réflexivité sur la société telle qu'elle se fait. Le risque comme expérience vécue et réfléchie peut ainsi au-delà d'une perspective catastrophiste élargir le champ du possible et du souhaitable. Le risque s'inscrit dans un espace démocratique de délibération et de mobilisation susceptible de conduire vers des solutions innovantes.

Dans la question du risque se jouent des paradigmes fondamentaux concernant des points nodaux d'articulation : articulation des niveaux d'action et de responsabilité collectifs et individuels, articulation des représentations et des comportements, articulation des temps - court et long - de l'action, articulation des acteurs et des voix.

Dire le risque est devenu une affaire collective aux mains d'experts scientifiques, juridiques mais aussi de la société civile. Elle est source de rhétoriques variées qui tour à tour avertissent, inquiètent et rassurent dans une même perspective de mise en visibilité-dicibilité de risques toujours plus invisibles.

Si l'espace public s'inscrit dans une théorie de la rationalité bureaucratique, il se délite néanmoins sous l'emprise des effets de la commercialisation. Force est de constater que les axiomes de régulation et de communication qui l'animent ne l'en dispensent pas. Le rapport dominants/dominés s'en voit reconfiguré à l'aune d'une critique sociale où prennent le pas, tant le contrôle numérique que la mondialisation. Patrick Chaskiel envisage une théorie analytique de la domination sous l'angle de la thématique publique qu'il nomme « la puissance communicationnelle » des risques technologiques à l'exclusion de l'approche procédurale des risques technologiques, contrepoids de leur approche strictement procédurale. Il nous invite à penser l'extension de cette capacité non perdue de thématisation en commun, appliquée à des objets qui peuvent être autant politiques, qu'économiques et techniques. Enfin, il envisage la tension entre un principe d'applicabilité (ressort d'une logique industrielle) et un principe d'implicabilité qui ne disparaît pas derrière la force du système 
technico-industriel mais au contraire se développe. La sphère de l'expérience vécue, réfléchie et discutée collectivement s'en trouverait renforcée. Dans cette acceptation conceptuelle, l'espace public serait investi comme un lieu propice à la solidarité, d'où un présupposé d'égalité. La posture critique de Patrick Chaskiel reconsidère les postulats d'Habermas. À titre d'exemple, il n'y aurait plus de «sphère de formation d'exigences universalistes ", mais un environnement propice aux discussions égalitaires. L'auteur applique son droit de réponse à un agir communicationnel qui complexifie, non pas le système en référence aux travaux de Luhmann, mais davantage la possibilité d'expansion du système. Le choix opéré par Patrick Chaskiel est de s'emparer de l'applicabilité et de la prévisibilité des nanotechnologies comme politique de financement de certains travaux scientifiques et labels. Il questionne en quoi les nanotechnologies entrent dans la thématique publique des risques? Il en conclut que la généralisation de nanosciences déconnecte le risque des thèmes environnementaux et sanitaires malgré des scandales comme les OGM ou encore le nucléaire. Néanmoins, un discours sociétal prend le pas sur cette tendance par l'annonce d'une politique "socio-tecnhinique nanotechnologique ", selon les termes de l'auteur. Démarche qui ne connaîtra pas de franc succès et se heurtera à la polémique. En effet, à la différence des contestations nées dans les années 1960 autour des effets et risques des produits toxiques, c'est désormais sur le bienfondé de l'existence des nanotechnologies que porte le débat et des dangers probables qui les accompagnent. La contestation se durcit au point de devenir radicale et de prendre une place à part entière sur la scène publique. L'émergence du principe de précaution traduit au plus près la politique de développement durable encadrant le risque par des chartes et débats conduisant à la mise en place de mesures, ce qui nôte rien au risque. La solution réside-t-elle davantage dans un devoir d'information et de conseil, voire dans l'harmonisation réglementaire européenne?

En partant du constat que la société occidentale est un entrelacs de sûreté et d'insécurité, d'un côté, fleurissent des mesures préventives et de l'autre un paysage réglementaire et normé en perpétuelle évolution avec, pour ne citer qu'un exemple, le principe de précaution. Le foisonnement d'accidents nucléaires, environnementaux ou sanitaires altèrent l'idéal sécuritaire faisant du risque une préoccupation centrale. Tant Ulrich Beck que Niklas Luhmann sont reconnus pour leurs travaux sur la question bien que divergents. Le premier voit la société industrielle fléchir sous le joug du risque, tandis que le second développe une «sociologie systémique du risque dans Ökologische Kommunikation " que Flavien Le Bouter explore dans son article. Le postulat est que malgré les avancées scientifiques, il n'est guère envisageable de cerner le risque dans toute sa complexité en tant quélément d'une société moderne liée à l'incertitude. Plus les systèmes sont complexes, plus l'incertitude croît et plus le risque augmente. L'expertise scientifique ne serait qu'une forme de consensus dans la société moderne. Selon les propos constructivistes de Niklas 
Luhmann analysés par Flavien Le Bouter « le savoir accroît l'incertitude et par là même le risque ". Loriginalité de Niklas Luhmann réside dans sa déconstruction de l'archétype conceptuel puisqu'il envisage le risque non plus à la lumière de la sécurité comme le font les médias ou bien les politiques, mais au prisme du danger que génère un événement. Entrent ici en scène les notions de responsabilité et de décision. Les nuances de l'article de Flavien Le Bouter reposent sur une sémantique du risque et les comparaisons contrastées qu'il fait entre Ulrich Beck et Niklas Luhmann. En marge de la temporalité et des systèmes politiques, le risque est ici repensé dans un espace public protestataire et revendicatif où la participation est illusoire. Cette vision est pessimiste, d'autant que l'approche systémique fait écho à un système, un environnement, et à un sujet, l'ensemble étant impliqué dans un mode opératoire à la différence d'Ulrick Beck.

En revenant sur le modèle théorique d'Ulrich Beck présenté dans La société $d u$ risque, la science devient incontournable et nécessaire, mais elle ne définit en rien la vérité. Fondée sur une approche industrielle qui touche davantage le nucléaire et les problèmes environnementaux, aucune réponse n'est de circonstance. L'autre acceptation du sujet serait de considérer le risque à l'aune de l'individualisation. Nous sommes ici dans deux conceptions singulières et divergentes que Paul Vancassel et Alain Calmès démontrent. Ils explorent les contours d'un fait de société aussi ancien et banal, qu'est le tabagisme. Devenu un fléau, il est combattu par des succédanés ou placebos (Vaporette) et par des mesures réglementaires tout en conservant ses prérogatives dans l'imaginaire collectif. La question du risque est adossée à celle de la gestion assurantielle et aux dommages causés, notamment, en matière de santé publique, voire d'addiction. En atteste également au niveau législatif la loi Evin des années 1990, suivie de l'interdiction de fumer dans les lieux publics et d'une loi du Parlement Européen en 2013. Toute la subtilité de la cigarette électronique, au sens des auteurs, est de contourner les circuits traditionnels de communication et de s'engager dans une "éco citoyenneté " d'un geste propre, sain et salutaire qui conserve sa connotation symbolique. De ce point de vue, le risque est évacué dans la mesure où les dimensions scientifiques, gestionnaires et politiques des risques collectifs industriels et technologiques, et leurs externalités négatives, ne sont pas en cause. Ici, c'est la logique sociétale qui prévaut pour la santé individuelle des usagers du tabac. Dans cette mesure, il n'y a ni expertise ni pragmatisme à la question du risque, mais plutôt une régulation et une normalisation du risque face à l'individualisme, voire à sa privatisation. La clef communicationnelle serait-elle celle de la responsabilisation d'acteurs autonomes face à leur prise de décision, liberté d'action et niveau d'information? Dans ce cas, les auteurs convergent-ils vers un care et self control responsable pour la prise en charge du risque?

Cette question concerne les organisations de santé soumises à une injonction. D'un côté, elles traitent l'aspect gestionnaire, de l'autre le volet médical. Le 
point commun est que le risque entourant la profession nécessite d'être proactif dans un environnement réglementé. Le near-miss, autour duquel Sylvie P. Alemanno axe son article se définit comme le " presqu'accident ». Il s'agit d'une sur-anticipation du risque traitée dans une approche compréhensive. Dans la filiation de l'interactionnisme symbolique, cette recherche prend en considération les acteurs et processus communicationnels où « l'instabilité des systèmes, saisie notamment par la théorie des systèmes sociaux développée par Niklas Luhmann (1984) » est à l'œuvre. Le near-miss potentialise, selon l'auteur, le système. Des logiques de confiance constituent les soubassements du near-miss et des artefacts communicationnels s'annoncent comme des formes du traitement des pratiques sanitaires. Elle analyse un dispositif particulier de prévention d'événements indésirables en situation médicale de soin, dispositif de vigilance sur-anticipatif couplé dans le cas retenu à une démarche managériale et à un principe de discussion déployé au sein de commissions dites de vigilance. Cette question concerne les organisations de santé soumises à une injonction de gestion de risques et de sécurité des patients. Le risque entourant la profession nécessite d'être pro-actif dans un environnement réglementé. Le near-miss, est traité dans une approche compréhensive, les logiques de confiance constituant les soubassements du near-miss : confiance construite, managée, verbalisée et techniquement orchestrée (dans un logiciel dédié).

À cet égard, le milieu médical est porteur de germes des risques et du changement organisationnel. En effet, des formes groupales de comportements sont identifiées par Catherine Loneux et Clément Gravereau dans le milieu hospitalier à l'occasion de la mise en place d'un nouveau système d'information hospitalier (SIH). Ce dernier engendre un risque de dérégulation des pratiques professionnelles, notamment en situation de risques psycho-sociaux (RPS) qui sont au cœur de cet article. Dans cet environnement, les auteurs s'inscrivent dans une approche du safe society où les normes, les discours et l'éthique sont examinés au regard des médias, relais d'opinion. En croisant TIC, RPS et SIH, les auteurs annoncent une " fracture de transition » dans les logiques d'action des acteurs médicaux et paramédicaux. La traçabilité et la responsabilité s'ajoutent aux relations interpersonnelles. Elles se renouvellent selon des formes émergentes, numériques, interactives mais dématérialisées, voire nomades. Si pour les soignants, la tâche est peu impactée, les acteurs du management sont concernés. Au-delà des contingences de changement organisationnel, c'est aussi une culture professionnelle qui se modifie avec les SIH en termes cognitifs et de temporalité. Les risques tournent autour des risques, de la sécurité, de la qualité ( $\mathrm{RO} 2 \mathrm{i}$ ) et du leitmotiv de la résistance au changement. Cette atomisation organisationnelle conduit à une crise, élément intimement lié au risque.

Aussi, dans un contexte de risques environnementaux et sanitaires, Françoise Bernard convoque le « continuum action, lien, sens, institution et imaginaire qui 
est au cœur de nombre de problématiques communicationnelles ». En reprenant les principales étapes de configuration de la question du risque en SHS et en SIC, elle rappelle l'ambiguïté et les métamorphoses d'un concept désormais surinvesti. Linstitution du risque est selon l'auteure qui cite volontiers les travaux de Cornelius Castoriadis une institution imaginaire dans laquelle deux aspects sont majeurs : les notions de participation et d'engagement. La participation comme pratique massivement convoquée peut « être étudiée dans sa dimension transformative de processus sociaux, politiques, économiques ", elle est solidaire de la notion tout aussi convoquée (notamment dans l'univers nord américain) d'empowerment qui renvoie tantôt à un levier de libération, tantôt à un principe de responsabilisation individuelle. L'engagement, autre notion clé, renvoie à la combinaison problématique d'idées et d'actes. Concernant leur articulation (au cœur de politiques d'information et de communication), Françoise Bernard s'inscrit dans une perspective résolument différente et, dans la foulée de Kurt Lewin, estime que " nos actions du présent sont davantage liées à nos actions du passé qu'aux idées nouvelles que nous aurions acquises sur la pertinence d'en changer ». Cette piste ouvre la voie d'une approche expérimentale qui déplace la relation entre action et signification et mobilise, au-delà de l'urgence, une temporalité longue. Le risque est omniprésent ; il est tant entrepreneurial, environnemental, médiatique, politique, sanitaire, scientifique, sociétal que technologique. Adossé au risque, le principe de précaution vient compléter l'approche. Il en est de même de la question de la responsabilité qui fait l'objet de nombreuses controverses sociotechniques dans les débats publics. Faut-il objectiviser le risque en le laissant aux mains d'experts scientifiques et juridiques ou bien faut-il laisser s'exprimer la part de subjectivité qui l'accompagne dans la société civile? L'auteur fait remarquer que l'appréhension du risque est le fait « d'observations longitudinales « c'est-à-dire qui ont besoin d'une temporalité longue même si l'urgence nécessite un traitement rapide. Cela corrobore le mouvement catastrophiste, alors que l'alternative du risque comme espace démocratique de délibération et de mobilisation peut conduire vers une issue concluante et innovante. À ce titre, la contribution de la communication est d'ouvrir des espaces de délibération publique où la participation, à la différence de l'approche pragmatique prend en considération le « sujet social " et son action. C'est donc au travers des pratiques relationnelles et du safe society que la publicisation et médiation du risque est rendue possible. Françoise Bernard fait appel aux notions d'empowerment et d'engagement comme " processus qui permet aux individus de prendre conscience de leur capacité d'agir et d'accéder à plus de pouvoir » (Zappi, 2013). En d'autres termes, c'est la capacité aux acteurs à se prendre en charge eux-mêmes face aux risques. On en revient à la question de l'autonomie, de la responsabilisation des acteurs-citoyens et de l'engagement dans les actes. Néanmoins, il n'y a pas nécessairement filiation 
entre nos idées et nos actes ce qui complexifie la relation au risque comme en attestent les travaux en psychologie sociale.

Il y a dans l'étude de Simona De Iulio et Susan Kovacs une vision programmatique de mise en adéquation de la prise de conscience et des comportements individuels avec les enjeux économiques liés aux modes de production générant des risques sanitaires. C'est à ce niveau que s'inscrit la question de la responsabilité qui s'exerce conjointement entre les parties prenantes sans déni ni parti pris. Cela relève d'un idéal même si des labels abondent en ce sens, pour ne citer que le PNA. Il en ressort une préoccupation de normalisation des dispositifs info pédagogiques où les discours de l'empowerment impliquent l'entreprise dans une promotion d'un agir collectif, tout en entretenant une gestion individuelle du risque qui relève d'une injonction paradoxale. Partant du principe selon lequel les situations à risque sont le fait "d'un réseau d'interactions socioculturelles ", Simona De Iulio et Susan Kovacs alimentent la fabrique de dispositifs d'information et de communication centrés autour des problématiques des risques alimentaires liés à l'enfance. Elles nourrissent leurs propos par une analyse de la rhétorique des processus discursifs en jeu dans la prise de conscience qui mènent à la responsabilisation. Sont mis à l'épreuve les supports pédagogiques et informatifs issus des établissements scolaires destinés aux élèves et leurs parents. Les logiques d'acteurs en cause sont tripartites. Il s'agit du rôle des programmes ministériels, des médiateurs que sont les établissements scolaires et de l'appropriation faite par les destinataires. Les éléments discursifs convoqués sont relatifs à un principe de causalité classique. Un ancrage pragmatique a été réalisé à Lille Métropole Communauté Urbaine et à la Fondation Louis Bonduelle. Principe qui pourrait être remis en cause par le scepticisme de Hume ou bien la distance critique de Kant. Lobjectif de l'étude est de réduire les risques, de construire une relation de confiance et d'interagir aux divers niveaux en cause, sachant que la temporalité varie et peut, potentiellement altérer les retombées escomptées, voire la crédibilité de l'action. Le dénominateur commun des campagnes d'information porte généralement sur des mises en scène caricaturales ou, a contrario, rebutantes, visant à inspirer dégoût, rejet ou moquerie des abus et dangerosités des produits alimentaires concernés. Toutefois, ni mises en garde, ni conduites à tenir ne sont concrètement énoncées. Seul le Programme National Nutrition Santé diffusé dans les restaurations scolaires s'empare des implicites des dangers et risques encourus par une mauvaise nutrition, sans pour autant engager le jeune public vers des normes et usages pour pallier les troubles alimentaires. $\mathrm{Si}$ le comportement est pédagogiquement abordé, le risque est laissé à la discrétion des documents de vulgarisation, souvent orientés vers les pratiques écologiques et les OGM, nitrates, etc.

Tandis que l'espace public est de plus en plus ouvert à la jurisprudence et à la médiatisation, Céline Pascual Espuny envisage une fracture de la société 
civile face à la montée généralisée du risque devenu omniprésent. Toutes les sciences s'emparent de cet objet communicationnel, scientifique, technique, juridique, etc. C'est sous l'angle de l'alerte que l'auteur envisage le risque. En déroulant le fil du cycle de vie du risque, de l'émetteur au destinataire, la notion de crise est convoquée et mise en scène sous la rampe des enjeux médiatiques. Qui lance l'alerte et à qui profite le risque ? Telles sont les questions posées par Céline Pascual Espuny. Les champs de la communication, de la sociologie et de la science politique émailleront l'article autour du point nodal de leader d'opinion. Le numérique est devenu le prolongement, voire la propagation du "virus » risque qui se répand dans les réseaux par les statuts partagés. Si les dynamiques ont changé, les leviers d'action et instruments du pouvoir aussi. Quelles prérogatives reste-il à l'appareil institutionnel normatif traditionnel, aux circuits internes dans une société civile où l'émetteur et sa crédibilité ne sont plus nécessairement des critères préventifs, palliatifs ou correctifs ? Les exemples alimentaires ne manquent pas d'illustrer cette hypothèse.

Le cas de l'eau à boire (C. Hervé Bazin) est proche, lié à une ensemble de scandales révélant la piètre qualité d'une boisson qui est normalement et fondamentalement source de vie. La mise en évidence du danger sanitaire et du risque invisible de ce produit quotidien enrôle des acteurs nombreux : associations de consommateurs, collectivités locales, industriels de l'eau, experts et médias. Selon Céline Hervé Bazin, « cette instrumentalisation des risques, des scandales et des expertises qui se contredisent bâtit la manufacture des risques et des valeurs ". Le risque alimentaire n'a jamais été l'objet d'une perception aussi importante alors qu'au fil des décennies les dispositifs de contrôle et de garantie ne cessent de se renforcer. Etrange paradoxe dans lequel se joue l'écart entre le risque réel et le risque perçu, écart dans lequel s'inscrit avec acuité la question de la confiance. Céline Hervé-Bazin plonge son lecteur dans l'interprétation de données statistiques émanant du CIEAU, du TNS, du SEDIF, de 60 millions de consommateurs, et de bien d'autres sources et jette le trouble sur l'eau consommée en France. La controverse est animée entre l'eau du robinet, l'eau de source et l'eau minérale. La déferlante médiatique trouve sa source, quant à elle, dans les scandales (taux de pesticides, emballages et recyclage, ressource rare, etc.). Autant d'éléments qui donnent de l'eau au moulin des détracteurs, et à leurs corolaires, les services marketing qui s'engouffrent dans la brèche pour colmater les fuites des rumeurs. L'auteur a mené une étude de terrain pour étayer les valeurs de l'imaginaire collectif associé à l'eau. Ses conclusions permettent de mieux comprendre les discours de ce qu'elle nomme «l'éco-blanchiment » de la communication, comme forme d'instrumentalisation du risque qui abreuve le corpus des interviews recueillies.

Enfin, après une lecture anthropologique puis une analyse des politiques sécuritaires, Gabrielle et Paul Rasse mettent en exergue la prévention des risques naturels et technologiques. Ils en arrivent au dénominateur commun de ce numéro 45 de Communication E̊ Organisation, à savoir que ce n'est pas 
le collectif qui l'emporte mais l'intérêt particulier. Si l'aléa et la conjonction sont les facteurs clefs du risque, la maîtrise des deux par des outils de gestion et d'actions de prévention permet de réduire le niveau de risque, et donc de minimiser le degré de catastrophe potentielle. Ces initiatives remontent aux années 1995 et 2003 à l'issue des accidents technologiques qui ont marqué la France (AZF), initialement avec des préoccupations de sécurité civile. C'est en s'appuyant sur un corpus d'articles du code de l'environnement que les auteurs évaluent le périmètre opérationnel de l'État en cas de prévention des risques, risques avérés ou de catastrophes. À cet égard, ils mentionnent des outils juridiques comme le PPRN (plan de prévention des risques naturels) et le PPRT (plan de prévention des risques technologiques) qui sont les références en matière de loi sur les risques. Le PPR émane des services déconcentrés de l'État et fait l'objet de débat public, voire concertation pour favoriser la compréhension des risques par la population et leur responsabilisation en cas de problème. Cela facilite la mise en œuvre de réglementations restrictives et favorise la politique urbaine dans les zones à risque. Au-delà de la prévention des risques, c'est une véritable préventologie qui émerge au bénéfice des acteurs qui tirent profit des peurs collectives et angoisses individuelles, notamment là où les juridictions officielles ont peu de prise (bidonvilles, zones de délinquance, zones défavorisées, etc.). 